\title{
Abdominal aortic pseudocoarctation
}

\author{
Roya Etemad-Rezai MD, Richard N. Rankin MB ChB
}

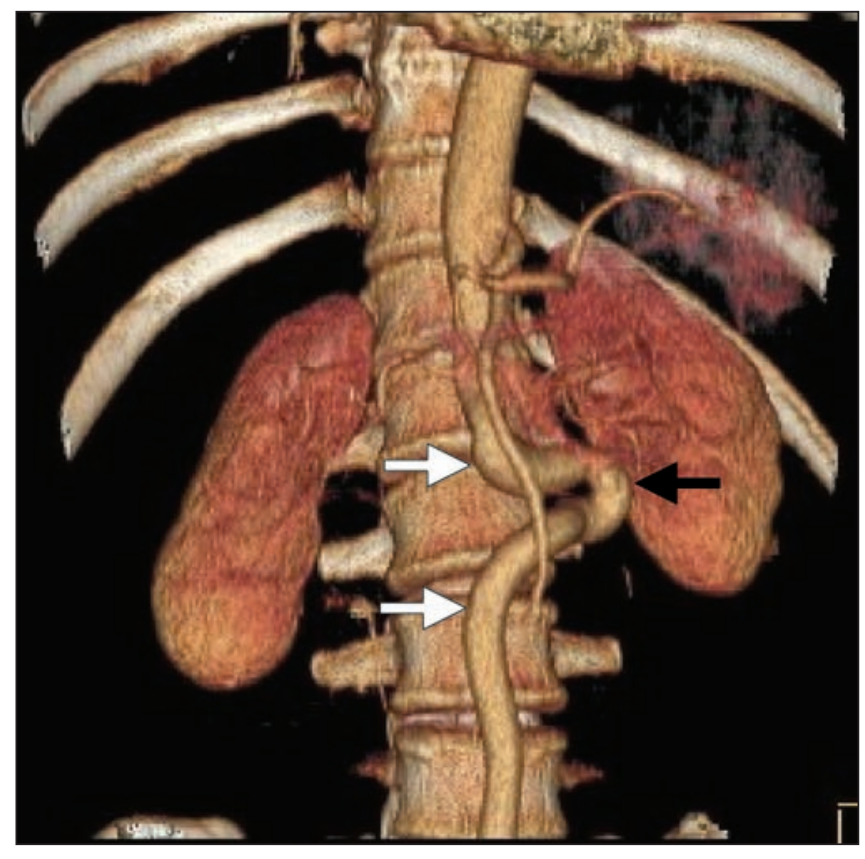

Figure 1: Volume-rendered reconstruction from computed tomography scans (anteroposterior view) showing abdominal aortic pseudocoarctation (black arrow). The extreme redundancy of the affected segment of aorta is clearly visible (white arrows).

The case: A 68-year-old woman with Waardenburg syndrome presented in 2005 with pain in her chest and back. A computed tomography scan of the thoraco-abdominal cavity showed an unusual aortic configuration. Her abdominal aorta at the $\mathrm{L} 2$ level deviated at right angles to the left to reach the left renal hilum, where it abruptly turned back through 180 degrees to reach the midline again at the L3 level. There it turned caudally and eventually bifurcated at the L5-S1 disc level into common iliac arteries (Figure 1, Figure 2, Figure 3). This configuration is consistent with abdominal aortic pseudocoarctation. Follow-up computed tomography scans in 2006 and 2007 showed no change in the aortic abnormality. The patient remains well with no cardiovascular abnormalities.

Abdominal aortic pseudocoarctation is very rare. To our knowledge, only 4 cases have been described to date, 1 of which involved cross-sectional imaging. ${ }^{1}$ It is similar in form

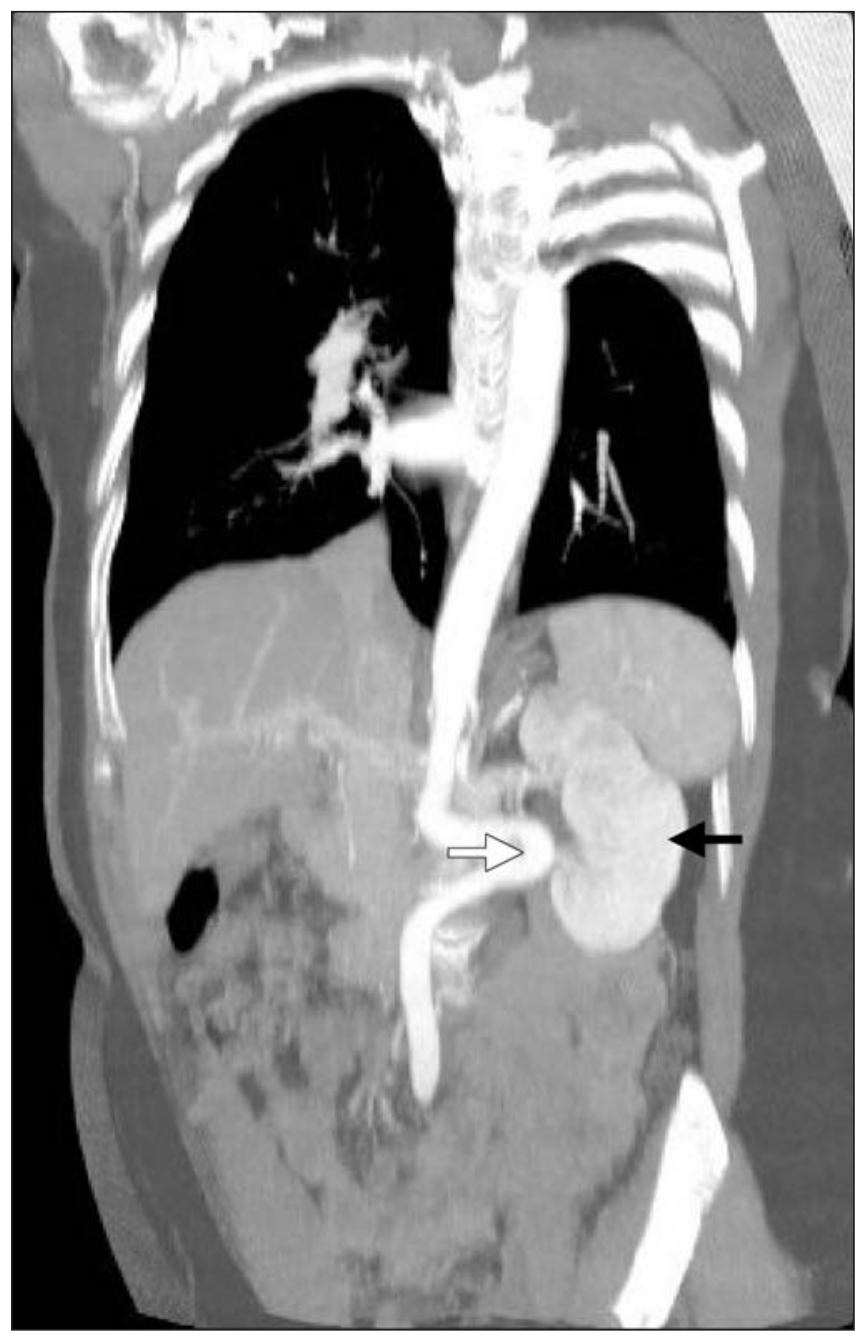

Figure 2: Coronal oblique reconstruction from abdominal computed tomography scans, showing the relationship of the abdominal aortic pseudocoarctation (white arrow) to the left kidney (black arrow).

to the more common condition of thoracic aortic pseudocoarctation, with marked redundancy of a segment of the aorta with no functional stenosis. The redundant tortuous segment does not have collateral circulation.

From the Department of Medical Imaging, University Hospital, London Health Sciences Centre, London, Ont. 


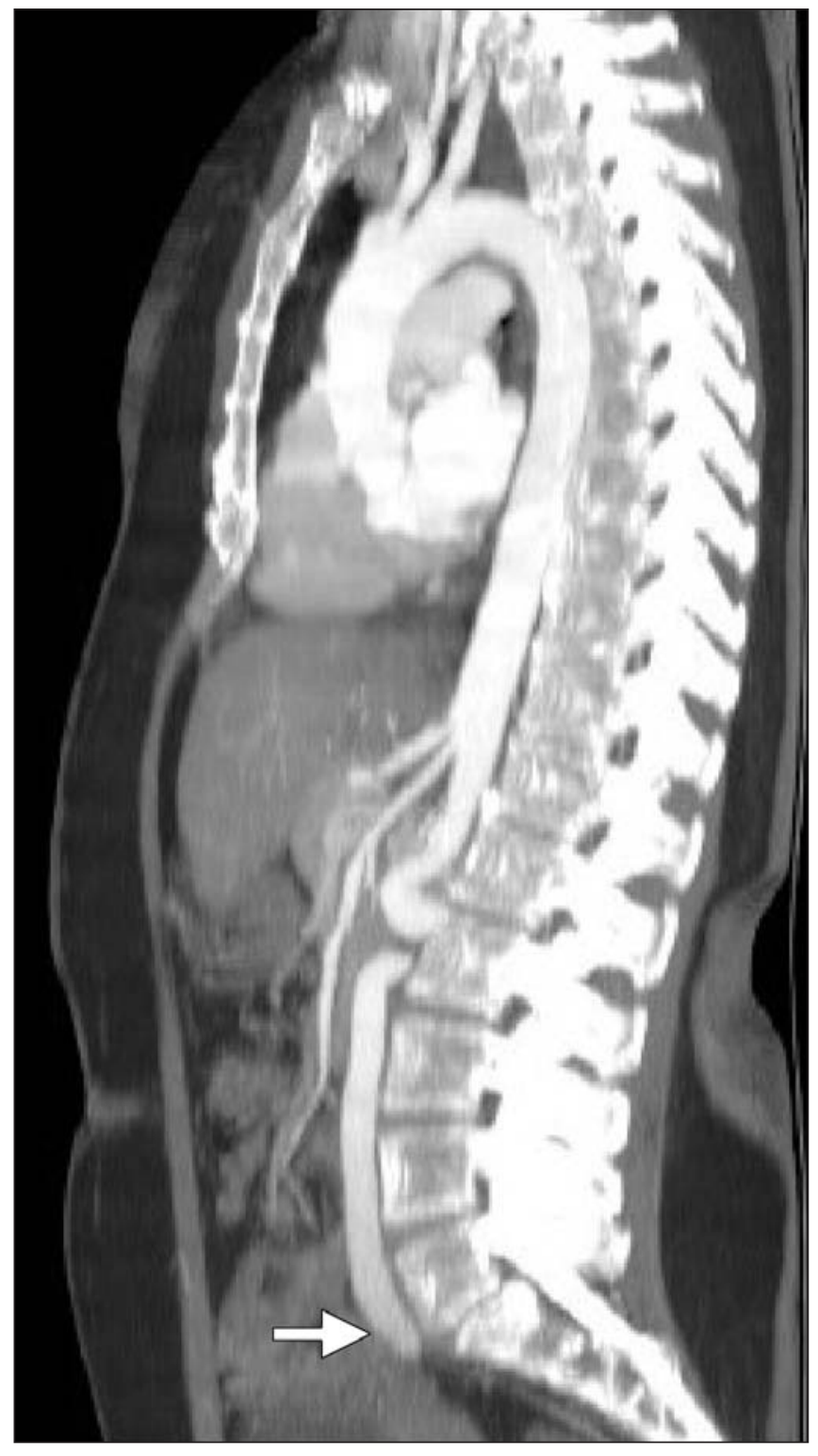

Figure 3: Sagittal reconstruction of a computed tomography scan of the thoraco-abdominal cavity. The abdominal aorta is normal in size, with no luminal obstruction down to and including its bifurcation at the L5-S1 level (arrow).
The abdominal aorta normally develops from the paired dorsal aortae by a process of fusion of the dorsal aortae at about the 25th day of fetal development. ${ }^{2}$ Disordered fusion is proposed as the cause of a range of vascular anomalies in this region of the body, from abdominal aortic coarctation or hypoplasia to dual-channel abdominal aorta, aortic interruption and pseudocoarctation. Some of these conditions may have associated symptomatology. All of them are rare, the last 3 exceedingly so. Recognition of such anomalies may increase as cross-sectional imaging and angiography become more widely used. Aortic pseudocoarctation should be recognized as a benign condition with no significant clinical associations.

This article has been peer reviewed.

Competing interests: None declared.

\section{REFERENCES}

1. Schellhammer F, von den Driesch P, Gaitzsch A. Pseudocoarctation of the abdominal aorta. Vasa 1997;26:308-10.

2. Arnot RS, Louw JH. The anatomy of the posterior wall of the abdominal aorta. Its significance with regard to hypoplasia of the distal aorta. S Afr Med J 1973;47:899-902.

Correspondence to: Dr. Roya Etemad-Rezai, Department of Medical Imaging, University Hospital, London Health Sciences Centre, 339 Windermere Rd., London ON N6A 5A5;

fax 519 663-8803; roya.etemadrezai@lhsc.on.ca

Teaching cases are brief case reports that convey clear, practical lessons. Preference is given to common presentations of important rare conditions, and important unusual presentations of common problems. Articles start with a brief summary (100 words) outlining the case and its relevance to a general audience. The case presentation follows (500 words maximum) as well as a discussion of the underlying condition (1000 words maximum). Generally, up to 5 references are permitted and visual elements (e.g., tables of the differential diagnosis, clinical features or diagnostic approach) are encouraged. Written consent from patients for publication of their story is a necessity and should accompany submissions. See information for authors at www.cmaj.ca. 